\title{
DIFFERENTIABLE INEQUALITIES AND THE THEORY OF CONVEX BODIES
}

\author{
BY \\ E. R. LORCH

\section{INTRODUCTION}

The definition of convexity requires that with every pair of points in a set $\mathfrak{M}$ the line segment joining them be also in $\mathfrak{M}$. Out of this seemingly naive concept issues a great river of mathematical wealth. First is the theory of the triangle inequality and with it the theory of linear spaces and their adjoint spaces. One may here substitute for the triangle inequality the bilinear inequality. At a more incisive level comes the theory of volumes and mixed volumes. In this range belong the Brunn-Minkowski theorem and the Minkowski inequalities. Beyond this, the directions of investigation multiply and cross-breeding with other domains takes place. Although the final results of the theory of convexity are particularly polished and striking, the path which to the present moment has led to them lacks transparence and directness. The fact that polyhedra play a dominant role in it illustrates this. For purposes of analysis, a polyhedron is often a very awkward object, with its sharp corners and over flat faces. The traditional definition of convexity is the cause of the difficulty. Since it lacks in finesse it is clear that its consequences frequently will be secured only by devious and peculiar methods.

The present paper presents a new definition of convexity. The theory which can be developed with its help is based on the highly precise propositions concerning homeomorphisms, homogeneous differentiable functions, and, particularly, positive definite quadratic forms. The development covers four chapters. The first is devoted to the introduction of the definition and the construction of the adjoint (polar, dual) surface. In the second, an analysis is made of bilinear inequalities of the form $\sum x_{i}^{*} y_{i} \leqq \psi\left(x^{*}\right) \phi(y)$. It is shown how to obtain all strict (differentiable) inequalities of this type. The methods not merely establish existence but show how these inequalities are to be computed.

The third chapter introduces a metric into certain classes of convex (or concave) surfaces. The metric space so defined is then made complete. Bilinear inequalities may be obtained for the elements of this space. A particular class (so-called "natural class") of surfaces is the class of closed surfaces. For this class it is shown that the surface of any body convex in the traditional sense may be approximated by surfaces convex in our sense. The

Presented to the Society, April 30, 1949 and June 17, 1950; received by the editors August 21, 1950 and, in revised form, January 21, 1951. 
fourth chapter gives applications to the theory of volumes and mixed volumes. By virtue of the preceding approximation theorem, this material is elaborated only for smooth surfaces. We develop here some new formulas which seem to be fundamental to the theory. Although it is as yet impossible to give an account of all Minkowski inequalities, it is probable that the present undertaking is a first step in that direction. In particular, it seems that the questions of mixed volumes are best handled with the techniques of tensor spaces and the exterior algebra. It is planned to pursue this matter in a future paper.

The paper calls for no specialized knowledge of any sort on the part of the reader. Each chapter is preceded by an introductory statement relative to its contents. Only one point of notation requires explanation. We omit indices of summation; thus $\sum x_{i} y_{i}$ means $\sum_{i=1}^{n} x_{i} y_{i}$, while $\sum \phi_{i j} z_{i} z_{j}$ and $\sum \phi_{i j} x_{j}$ mean $\sum_{i, j=1}^{n} \phi_{i j} z_{i} z_{j}$ and $\sum_{j=1}^{n} \phi_{i j} x_{j}$ respectively. We make no attempt in the text to introduce remarks of historical content. The specialist will easily discern what contributions may have been made here. For a detailed statement of the development of this subject we refer to the very excellent treatment, Konvexe Körper, given by Bonnesen and Fenchel. At about the time the material of the first two chapters was read to the Society, an interesting note on this subject was published by Fenchel (On conjugate convex functions, Canadian Journal of Mathematics vol. 1 (1949) pp. 73-77). There are many points of similarity between the results of Fenchel and some of those treated in these chapters. However, closer examination shows that the spirit of the two investigations is different. For example, Fenchel does not give constructive methods for "computing" inequalities. For Fenchel, the notation of convex region is dominant whereas for us, it is that of homeomorphism. In view of the fact that we wished to give a unified theory of convexity which may be applied to the theory of mixed volumes, we have carried through the development exclusively from our own point of view although we indicate in the text the connection with the work of Fenchel.

\section{ThE NOTION OF CONVEXITY FOR DIFFERENTIABLE SURFACES}

1. Differentiable surfaces. We are concerned with a certain class of surfaces in a real $n$-dimensional space. Coordinates in this space will be denoted by $x_{1}, \cdots, x_{n}$ and the point $\left(x_{1}, \cdots, x_{n}\right)$ will frequently be referred to as a vector, and will be denoted by $x$.

Consider a function $\phi(x)=\phi\left(x_{1}, \cdots, x_{n}\right)$. Let $\phi$ be defined on a rayspace $\Omega$, that is, an open connected set such that if $x \in \Omega$, so does $c x$ where $c$ is a positive scalar. We further assume that $\Omega$ does not contain the origin. Typical domains $\Omega$ are: (1) the entire space excepting the point $x=0 ;(2)$ the set of all points $\left(x_{1}, \cdots, x_{n}\right)$ for which $x_{i}>0, i=1, \cdots, n$. We assume that $\phi(x)$ is positive on $\Omega$ and that it is positively homogeneous of degree 1 ; that is, for $t>0, \phi(t x)=t \phi(x)$. Finally, it will be assumed that $\phi(x)$ possesses 
a sufficient number of derivatives to permit of the operations on it indicated in the sequel. Our results would not be weakened essentially if we confined ourselves to real analytic functions.

The equation $\phi(x)=c, c>0$, defines a surface $\Im_{c}$. The surfaces $\Im_{c}$ form a homothetic family obtained by magnification of $\mathfrak{S}_{1}$ at the origin by the factor $c$.

Let $r$ be any real number except zero, $r \neq 0$. Write $G(x)=r^{-1}[\phi(x)]$ r. Then $G(x)=c^{\prime}$ represents the same family of surfaces. One may note in passing that if $r>0, c^{\prime}$ assumes positive values; if $r<0$, then $c^{\prime}<0$ also. For any $r$, an increase in the value of $c^{\prime}$ produces a magnification of the corresponding surface. In the future, we shall always assume that the equation of the homothetic family of surfaces with which we are dealing is of the form

$$
G(x)=c
$$

where $G(x)$ is homogeneous of degree $r \neq 0$ and $c$ is a constant such that $r c>0$.

2. The associated transformation. Each function $G(x)$ in (1) defines a transformation of fundamental importance to what follows. This transformation is given by the equations

$$
x_{i}^{*}=G_{i}\left(x_{1}, \cdots, x_{n}\right), \quad i=1, \cdots, n,
$$

where $G_{i}\left(x_{1}, \cdots, x_{n}\right)$ represents $\partial G(x) / \partial x_{i}$. Thus to every point $x$ $=\left(x_{1}, \cdots, x_{n}\right)$ in $\Omega$ is associated another point $x^{*}=\left(x_{1}^{*}, \cdots, x_{n}^{*}\right)$ which we shall locate in a second $n$-dimensional space. The totality of these points $x^{*}$ will be denoted by $\Omega^{*}$.

We now make the assumption that the degree of homogeneity, $r$, of $G(x)$ is not 1: $r \neq 1$. Then the degree $r-1$ of $G_{i}(x)$ is not 0 and hence one may establish easily the fact that $\Omega^{*}$ possesses the properties of a ray-space except that it may not be open.

3. The associated quadratic form. The fundamental transformation (2) has a Jacobian $\left|G_{i j}(x)\right|$ where $G_{i j}(x)$ represents $\partial^{2} G(x) / \partial x_{i} \partial x_{j}$. This Jacobian is the Hessian of $G(x)$. We introduce now the fundamental quadratic form

$$
(r-1) \sum_{i, j} G_{i j}(x) z_{i} z_{j}
$$

where $z=\left(z_{1}, \cdots, z_{n}\right)$ is an arbitrary vector.

4. The adjoint surface. If we start with a family of surfaces $G(x)=c$, we may construct a second family $F\left(x^{*}\right)=b$ in a manner presently to be described. The new family is the adjoint of the old. In the terminology of the theory of convex bodies, the second surface defines a body which is the polar of the first.

We assume henceforth that the quadratic form (3) is nonsingular for any $x \in \Omega$.

From this follows that the Jacobian of (2) is not zero. Hence the equations (2) define a local homeomorphism between $\Omega$ and $\Omega^{*}$. 
We assume that the local homeomorphism above actually is a homeomorphism in the large.

Conditions on $\Omega$ and $G(x)$ will be given presently which insure that this last assumption is satisfied. If we invert equations (2) we obtain

$$
x_{i}=F_{i}\left(x_{1}^{*}, \cdots, x_{n}^{*}\right), \quad i=1, \cdots, n .
$$

It should be noted that the notation $F_{i}\left(x^{*}\right)$ may not as yet be taken to indicate that there is a function $F\left(x^{*}\right)$ whose partial derivative with respect to $x_{i}^{*}$ is $F_{i}\left(x^{*}\right)$. This does happen to be the case but needs proof.

First note that $\partial F_{i} / \partial x_{j}^{*}=\partial F_{j} / \partial x_{i}{ }^{*}$. For, consider the matrix $\left\|G_{i j}(x)\right\|$ of the quadratic form (3), and the matrix $\left\|F_{i j}\left(x^{*}\right)\right\|$ obtained from (4) by setting $F_{i j}=\partial F_{i} / \partial x_{j}{ }^{*}$. The product of these two matrices is the identity matrix. Since $\left\|G_{i j}(x)\right\|$ is symmetric, so is $\left\|F_{i j}\left(x^{*}\right)\right\|$.

Also, since $G_{i}(x)$ is homogeneous of degree $r-1$, then $F_{i}\left(x^{*}\right)$ is homogeneous of degree $(r-1)^{-1}$. Using Euler's theorem on homogeneous functions it is easy to see that if we define $F\left(x^{*}\right)=r^{-1}(r-1) \sum x_{i}{ }^{*} F_{i}\left(x^{*}\right)$, then $\partial F\left(x^{*}\right) / \partial x_{i}^{*}=F_{i}\left(x^{*}\right)$. Thus $F_{i}$ in (4) represents a partial derivative.

Let us write $1 / r+1 / r^{\prime}=1$. Then we see immediately that $F\left(x^{*}\right)$ is homogeneous of degree $r^{\prime}, r^{\prime} \neq 0,1$. Also, by Euler's theorem we have $\sum x_{i} x_{i}{ }^{*}$ $=\sum G_{i}(x) \cdot x_{i}=r G(x)$ and also $\sum x_{i} x_{i}{ }^{*}=F_{i}\left(x^{*}\right) \cdot x_{i}{ }^{*}=r^{\prime} F\left(x^{*}\right)$. Hence $r G(x)$ $=r^{\prime} F\left(x^{*}\right)$. Furthermore, since $(r-1)\left(r^{\prime}-1\right)=1$, we have $(r-1)\left\|G_{i j}(x)\right\|$ $\cdot\left(r^{\prime}-1\right)\left\|F_{i j}\left(x^{*}\right)\right\|=\left\|\delta_{i j}\right\|$. Hence the quadratic form (3) and the quadratic form $\left(r^{\prime}-1\right) \sum F_{i j}\left(x^{*}\right) z_{i} z_{j}$ are both positive, or both indefinite. Since $r G(x)=\phi^{r}(x)>0$ and since $(r-1) \sum G_{i j}(x) x_{i} x_{j}=r(r-1)^{2} G(x)$, the above quadratic forms can never be negative.

We have said above that the family of surfaces $F\left(x^{*}\right)=b$ is adjoint to the family $G(x)=c$. More precisely, we shall say that the surface $r^{\prime} F\left(x^{*}\right)=1$ is the adjoint of the surface $r G(x)=1$. It is clear that the relationship is reciprocal.

5. The definition of convexity. We are now in a position to define convexity. A principal thesis of this paper is that the classic definition of convexity must be discarded in favor of another which may seem slightly more restrictive at first but which leads to a much richer mathematical development. In fact, we shall see essentially that our present definition involves much of the structure which is associated with definite quadratic forms, homogeneous differentiable functions, and homeomorphisms.

DEFINITION 1. Let a homothetic family of surfaces be defined in some rayspace $\Omega$ by the equation $G(x)=c$. Suppose the degree $r$ of homogeneity of $G(x)$ satisfies $r>1$. Then we shall say that the surfaces are convex throughout $\Omega$ if the quadratic form $(r-1) \sum G_{i j}(x) z_{i} z_{j}$ is positive definite for every $x$ in $\Omega$.

The geometric significance of this concept of convexity (curvature of the surface) will be treated presently. In a similar way we define concavity.

DeFINITION 2. Let a homothetic family of surfaces be defined in some ray- 
space $\Omega$ by the equation $G(x)=c$. Suppose the degree $r$ of homogeneity of $G(x)$ satisfies $r<1, r \neq 0$. Then we shall say that the surfaces are concave throughout $\Omega$ if the quadratic form $(r-1) \sum G_{i j}(x) z_{i} z_{j}$ is positive definite for every $x$ in $\Omega$.

It is clear from $\$ 4$ that a surface is convex or concave if and only if the adjoint surface has the same property.

We shall now show how these definitions were conceived Consider the case of convexity. Let $x$ be a fixed point on the surface $G(x)=c$. Let $u$ be an arbitrary vector. Then we may express $u$ as the sum of two vectors $v$ and $w$ in a unique fashion, $u=v+w$, where $v$ is a scalar multiple of $x, v=t x$, and $w$ is parallel to the hyperplane which is tangent to the surface at the point $x$. Thus for $w$ we have $\sum G_{i}(x) w_{i}=0$.

Next, for an arbitrary vector $z$, we have

$$
G(x+z)=G(x)+\sum G_{i}(x) z_{i}+\frac{1}{2} \sum G_{i j}(x) z_{i} z_{j}+R,
$$

where $R$ denotes the remainder term of the Taylor expansion. If $z$ lies in the tangent plane, then $\sum G_{i}(x) z_{i}=0$. Let $z$ be small. Suppose furthermore that our surface is convex in the classic sense-for example, the surface might be the boundary of a convex body. Then it is clear that $G(x+z) \geqq G(x)$. Hence it follows that $\sum G_{i j}(x) z_{i} z_{j} \geqq 0$ for all small $z$ lying parallel to the tangent hyperplane.

Now suppose that $\sum G_{i j}(x) z_{i} z_{j}>0$ for all vectors $z \neq 0$ which are parallel to the tangent hyperplane. Let $u$ be arbitrary and write $u=t x+z$. Then

$$
\begin{aligned}
\sum G_{i j}(x)\left(t x_{i}+z_{i}\right)\left(t x_{i}+z_{j}\right)= & t^{2} \sum G_{i j}(x) x_{i} x_{j}+2 t \sum G_{i j}(x) x_{i} z_{j} \\
& +\sum G_{i j}(x) z_{i} z_{j} .
\end{aligned}
$$

The first term equals $t^{2} r(r-1) G(x)$ and is positive unless $t=0$. The last term is positive also for $z \neq 0$ by the hypothesis at the beginning of this paragraph. And $\sum G_{i j}(x) x_{i} z_{j}=(r-1) \sum G_{i}(x) z_{i}=0$ by the symmetry of the bilinear form and by Euler's theorem. Hence with our present hypothesis, $\sum G_{i j}(x) u_{i} u_{j}$ is a positive definite form. This discussion indicates how the present definition of convexity was developed.

We show briefly that the above definition implies classical convexity. Consider a convex body bounded by a differentiable surface $G(x)=c$. In this case $\Omega$ consists of the entire space except $x=0$. If $x$ is as before and $z$ lies in the hyperplane tangent at $x, x+z$ is an arbitrary point in the tangent hyperplane. Then

$$
G(x+z)-G(x)=\int_{0}^{1}[d G(x+\rho z) / d \rho] d \rho=\int_{0}^{1} \sum G_{i}(x+\rho z) z_{i} d \rho .
$$

Since the integrand is zero for $\rho=0$ and since its derivative with respect to $\rho, \sum G_{i j}(x+\rho z) z_{i} z_{j}$, is positive if $z \neq 0$, we see that $G(x+z)>G(x)$. Hence our 
definition of convexity implies classical convexity.

The relation between the type of convexity here discussed and the geometry of the surface $\phi(x)=1$ has been pointed out by F. Steinhardt and may be set down in a few lines.

Since $G_{i j}=\phi^{r-2}\left[(r-1) \phi_{i} \phi_{j}+\phi \phi_{i j}\right]$, we have

$$
(r-1) \sum G_{i j}(x) z_{i} z_{j}=(r-1) \phi^{r-2}(x)\left[(r-1)\left[\sum \phi_{i}(x) z_{i}\right]^{2}+\phi(x) \sum \phi_{i j}(x) z_{i} z_{j}\right] .
$$

Thus " $\phi(x)=1$ is convex in our sense" implies and is implied by " $\sum \phi_{i j}(x) z_{i} z_{j}$ is non-negative definite and $\left\|\phi_{i j}(x)\right\|$ has rank $n-1$." It is well known that the latent roots of the matrix $\left\|\phi_{i j}\right\|$ are in addition to the number zero, the principal radii of curvature of the surface adjoint (polar) to $\phi(x)=1$. Thus the convexity of $\phi(x)=1$ is equivalent to the positive character of the principal radii of curvature of its adjoint surface. Now $\phi(x)=1$ is convex if and only if its adjoint surface $\psi\left(x^{*}\right)=1$ is convex. Hence " $\phi(x)=1$ is convex" implies and is implied by "the principal radii of curvature of $\phi(x)=1$ are all positive."

6. Properties of the quadratic form. Let $\phi(x)$ be the function introduced earlier and consider the matrix $\left\|\phi_{i j}(x)\right\|$. Since $\sum \phi_{i j}(x) x_{j}=0$ by Euler's theorem, the form $\sum \phi_{i j}(x) z_{i} z_{j}$ is singular. Let $G(x)=r^{-1} \phi^{r}(x)$. Then since

$$
G_{i j}(x)=(r-1) \phi^{r-2}(x) \phi_{i}(x) \phi_{j}(x)+\phi^{r-1}(x) \phi_{i j}(x)
$$

we have $\left\|G_{i j}\right\|=(r-1) \phi^{r-2}\left\|\phi_{i} \phi_{j}\right\|+\phi^{r-1}\left\|\phi_{i j}\right\|$. For convenience we have omitted the argument $x$. Now note that if $u=t x+z$ is an arbitrary vector, where $z$ lies in the tangent hyperplane, then $\sum \phi_{i} \phi_{j} u_{i} u_{j}=\left(\sum \phi_{i} u_{i}\right)^{2}=\left(t \sum \phi_{i} x_{i}\right.$ $\left.+\sum \phi_{i} z_{i}\right)^{2}=t^{2} \phi^{2}$. Also $\sum \phi_{i j} u_{i} u_{j}=\sum \phi_{i j} z_{i} z_{j}$. Thus $(r-1) \sum G_{i j} u_{i} u_{j}=(r-1)^{2} t^{2} \phi^{r}$ $+(r-1) \phi^{r-1} \sum \phi_{i j} z_{i} z_{j}$. Now suppose that for a fixed $\phi$ and with $r>1$, this quadratic form is positive. Then certainly it is positive if $t=0$ and $z \neq 0$. Thus $\sum \phi_{i j} u_{i} u_{j}$ is positive for $u$ in the tangent hyperplane. Now, keeping $\phi$ fixed, let $r<1$. Then $(r-1)^{2} t^{2} \phi^{r}>0$ if $t \neq 0$ while $(r-1) \sum \phi_{i j} z_{i} z_{j}<0$ if $z \neq 0$. Thus as $r-1$ varies from a positive to a negative value, the index of the quadratic form changes from $n$ to 1 .

Suppose now that for a fixed $\phi$ and with $r<1$, the form $(r-1) \sum G_{i j} u_{i} u_{j}$ is positive for $u \neq 0$. Then $\sum \phi_{i j} z_{i} z_{j}<0$ for $z \neq 0$. If we choose $r$ so that $r>1$, it is clear that the index of the form $(r-1) \sum G_{i j} u_{i} u_{j}$ becomes 1 just as in the previous case.

Let us write $r^{\prime} F\left(x^{*}\right)=\psi^{\prime \prime}\left(x^{*}\right)$. We have $\left\|F_{i j}\left(x^{*}\right)\right\| \cdot\left\|G_{i j}(x)\right\|=\left\|\delta_{i j}\right\|$. Now $F_{i j}=\psi^{\prime-2} \cdot\left(\left(r^{\prime}-1\right) \psi_{i} \psi_{j}+\psi \psi_{i j}\right)$ with a similar expression for $G_{i j}$. Suppose that $x$ be so chosen that $\phi(x)=1$. Then $\psi\left(x^{*}\right)=1$. Also $x_{i}^{*}=\phi^{r-1}(x) \phi_{i}(x)=\phi_{i}(x)$ and $x_{i}=\psi_{i}\left(x^{*}\right)$. Thus we have

$$
\left\|F_{i j}\right\| \cdot\left\|G_{i j}\right\|=\left\|\psi_{i} \psi_{j}\right\| \cdot\left\|\phi_{i} \phi_{j}\right\|+\left\|\psi_{i j}\right\| \cdot\left\|\phi_{i j}\right\|=\left\|\delta_{i j}\right\| .
$$

In obtaining this expression use has been made of the fact that $\left\|\psi_{i} \psi_{j}\right\| \cdot\left\|\phi_{i j}\right\|$ $=0$ since $\sum_{k} \psi_{i} \psi_{k} \phi_{k j}=\psi_{i} \sum \phi_{k j} \cdot x_{k}=0$. Furthermore $\sum_{k} \psi_{i} \psi_{k} \phi_{k} \phi_{j}=\psi_{i} \phi_{j} \sum \phi_{k} \cdot x_{k}$ 
$=\psi_{i} \phi_{j} \phi=\psi_{i} \phi_{j}$. Hence $\left\|\psi_{i} \psi_{j}\right\| \cdot\left\|\phi_{i} \phi_{j}\right\|=\left\|\psi_{i} \phi_{j}\right\|=\left\|x_{i} x_{j}^{*}\right\|$. The latter matrix is easily seen to be of rank 1 . The matrix $\left\|\psi_{i j}\right\|$ is not the reciprocal of $\left\|\phi_{i j}\right\|$ but satisfies the equation

$$
\left\|\psi_{i j}\right\|\left\|\phi_{i j}\right\|=\left\|\delta_{i j}\right\|-\left\|\psi_{i} \phi_{j}\right\| .
$$

Finally, we shall show that if the union of $\Omega$ and the origin $x=0$ is a convex set, then for a convex family $G(x)=c$, the local homeomorphism between $\Omega$ and $\Omega^{*}$ defined by (2) is actually one-to-one and hence is a homeomorphism in the large. It must be added that $\Omega^{*}$ need not be convex even though $\Omega$ is. Thus in our treatment we have a homeomorphism but the domains may not be convex; in that of Fenchel, the domains are always convex but in general there is no homeomorphism.

To prove our statement, assume that the equations (2) transform the vectors $x$ and $y$ into the same point. Consider the expression $G(\theta x+(1-\theta) y)$, $0 \leqq \theta \leqq 1$. The derivative of this function of $\theta$ has equal values at $\theta=0$ and $\theta=1$. Hence the second derivative $\sum G_{i j}(\theta x+(1-\theta) y)\left(x_{i}-y_{i}\right)\left(x_{j}-y_{j}\right)$ vanishes for a suitable $\theta$. This means that $x-y=0$. In this proof it has been assumed that $\theta x+(1-\theta) y$ does not pass through the origin. This exceptiona case presents no difficulties.

\section{The FUndamental INEQUALITIES}

1. Statement of problem. We shall study in what follows questions concerning inequalities of the type $\sum x_{i} y_{i} \leqq \phi(x) \psi(y)$. Examples of such inequalities are furnished by the Hölder and the Cauchy-Schwarz inequalities. The inequality between geometric and arithmetic means is also of this class, as we shall see later, with the sign reversed. These inequalities for the case of convex bodies where treated by Minkowski. The recent work of Fenchel gives a solution to some of these problems for more general situations of the type we treat. However, since Minkowski's and Fenchel's methods are geometrical rather than analytical, it is understandable that they do not yield methods for the actual derivation of these inequalities, for what one may call their computation; they merely have existential force. Our methods show that it is sufficient to invert a transformation of one $n$-space into another to obtain an inequality. This means that the computation is as difficult to carry out in explicit form as this inversion is. One should note that, in addition, we require whatever calculations are necessary to ascertain whether a given quadratic form is positive or not. This may be of considerable difficulty in some cases. Theorem 1 gives a uniqueness property for inequalities. Its proof is an extension of a similar one given by the author for the Cauchy-Schwarz inequality (The Cauchy-Schwarz inequality and selfadjoint spaces, Ann. of Math. vol. 46 (1945) pp. 468-473).

We consider the case of convexity. Let $G(x)=c$ denote a convex family of surfaces as defined in chapter I. We assume furthermore that the union of 
the origin and of $\Omega$ is a convex point set. Suppose that $r>1$ and that $x_{i}^{*}$ $=G_{i}(x)$. Let $x$ and $y$ be arbitrary vectors in $\Omega$. Let $\phi(x)$ represent a function as yet unknown, of which nothing is assumed except that $\phi(x)>0$ for all $x$. We specifically underline the fact that $\phi(x)$ does not represent the function defined in chapter I. The fundamental problem of this chapter is to determine all functions $\phi(x)$ which satisfy the extremum property: For every $x$,

$$
\max _{y} \frac{\sum x_{i}^{*} y_{i}}{\phi(y)}=\frac{\sum x_{i}^{*} x_{i}}{\phi(x)} \text {. }
$$

The extremum property in (10) could equally well have been stated as an inequality. For this reason, we shall frequently refer to (10) as an inequality.

2. Solution of problem. The above problem has a unique solution which is given in

THEOREM 1. Let $G(x)=c$ denote a convex family of surfaces. Let the quantities $x, y, x^{*}$, and $\phi(x)$ be as defined above. Then the equation (10) has solutions and these are given precisely by the formula

$$
\phi(x)=k\left(\sum x_{i}^{*} x_{i}\right)^{1 / r}
$$

where $k$ is an arbitrary positive constant.

Furthermore, the inequality defined by (10) is strict; that is, for the value of $\phi(x)$ just given, and for a given $x$, the only solution $y$ of (10) is $y=t x$ where $t>0$.

This implies that the $\phi(x)$ here introduced is the same as that which appears in chapter I.

The proof will be based on some lemmas.

Lemma 1. Any solution $\phi(x)>0$ of (10) is positively homogeneous of degree 1 ; that is, $\phi(c x)=c \phi(x)$ for $c>0$.

Proof. Let $c>0$. Consider a fixed $x$. Then from (10) we see that $\phi(x) \sum x_{i}^{*}\left(c x_{i}\right) \leqq \phi(c x) \sum x_{i}^{*} x_{i}$. Hence $c \phi(x) \leqq \phi(c x)$. In a similar fashion we have that $\phi(c x) \sum\left(c x_{i}\right)^{*} x_{i} \leqq \phi(x) \sum\left(c x_{i}\right)^{*}\left(c x_{i}\right)$, hence $\phi(c x) \leqq c \phi(x)$. This establishes the lemma.

By virtue of Lemma 1 we may restrict ourselves to such values of $x$ for which $\sum x_{i}^{*} x_{i}=1$. Then in order to establish the theorem, it will suffice to show that for $x$ restricted in this manner, $\phi(x)$ is a constant. Since $\phi(x)$ is positive by hypothesis, this constant is positive.

Lemma 2. Any solution $\phi(x)$ of (10) is constant on the surface $\sum x_{i}{ }^{*} x_{i}=1$.

The proof will be established in the following way. Consider two points on $\sum x_{i}{ }^{*} x_{i}=1$. Join these points by a curve lying on that surface and which is arbitrary except that it must be differentiable. Let the curve be $y=y(t)$ where $y(0)=x$. Write $\phi(y(t))=\xi(t)$. We shall show that $\xi(t)$ is differentiable 
at $t=0$ and $\xi^{\prime}(0)=0$. Since $x$ is arbitrary, $\xi(t)=k$.

From (10) we have $\xi(0) \sum x_{i}^{*} y_{i} \leqq \xi(t) \sum x_{i}^{*} x_{i}=\xi(t)$. Now for $t$ small and positive,

$$
\left[\sum x_{i}^{*} y_{i}-1\right] \xi(0) t^{-1} \leqq(\xi(t)-\xi(0)) t^{-1} .
$$

Also since $\phi(y) \sum x_{i} y_{i}{ }^{*} \leqq \phi(x) \sum y_{i} y_{i}{ }^{*}$, we have

$$
[\xi(t)-\xi(0)] t^{-1} \leqq\left[\left(\sum x_{i} y_{i}^{*}\right)^{-1}-1\right] \xi(0) t^{-1} .
$$

The above expressions give information on the derivative of $\xi(t)$ at $t=0$. We shall show that

$$
\lim _{t \rightarrow 0}\left[\sum x_{i}^{*} y_{i}-1\right] t^{-1}=0 ; \quad \lim _{t \rightarrow 0}\left[\left(\sum x_{i} y_{i}^{*}\right)^{-1}-1\right] t^{-1}=0 .
$$

To this end it is necessary to show that $\sum x_{i}{ }^{*} \dot{y}_{i}(0)=0$ and $\sum x_{i} \dot{y}_{i}{ }^{*}(0)=0$ where the dot indicates differentiation.

By virtue of the equation $\sum y_{i}{ }^{*}(t) y_{i}(t)=1$, these two conditions are equivalent. The latter equation may be written in the form $r G(y(t))=1$. If we differentiate the latter we obtain $\sum G_{i} \dot{y}_{i}=0$, hence, setting $t=0, \sum x_{i}{ }^{*} \dot{y}_{i}(0)=0$. This shows that $\xi^{\prime}(0)=0$ and hence establishes the lemma.

Suppose that on $\sum x_{i}^{*} x_{i}=1, \phi(x)=k$. Then since $\phi(x)$ is homogeneous of order 1 , it is easy to see that for arbitrary $x, \phi(x)=k\left(\sum x_{i}{ }^{*} x_{i}\right)^{1 / r}$. For let $c$ be such that $\sum(c x)_{i}^{*}\left(c x_{i}\right)=1$. Then $\phi(x)=\phi\left(c^{-1} \cdot c x\right)=c^{-1} \phi(c x)=c^{-1} k$. On the other hand, since $x_{i}{ }^{*}=G_{i}(x)$ is homogeneous of degree $r-1,(c x)_{i}{ }^{*}=G_{i}(c x)$ $=c^{r-1} x_{i}{ }^{*}$ and hence $\sum(c x)_{i}{ }^{*}\left(c x_{i}\right)=c^{r} \sum x_{i}^{*} x_{i}=1$. This discussion proves that any solution $\phi(x)$ must be of the type indicated in the theorem. We now proceed to establish the converse of this statement.

Lemma 3. Let $\phi(x)=k\left(\sum x_{i}{ }^{*} x_{i}\right)^{1 / r}$ with $k>0$. Then $\phi(x)$ satisfies the condition (10). Furthermore the inequality defined by (10) is strict in the sense indicated by the theorem.

Let $x$ be chosen and held fixed. Consider an arbitrary $y$. Then $y=t x+z$ where $z$ is parallel to the hyperplane tangent at $x$. Since $x_{i}^{*}=G_{i}(x)$, we have $\sum x_{i}{ }^{*} y_{i}=t \sum G_{i}(x) x_{i}+\sum G_{i}(x) z_{i}=t \sum x_{i}{ }^{*} x_{i}$. If $t \leqq 0$, certainly (10) is verified. Now suppose $t>0$. If $z=0$, then we have an equality in (10). If $z \neq 0$ then $G(t x+z)>G(t x)$. For consider the function of $\theta, f(\theta)=G(t x+\theta z), 0 \leqq \theta \leqq 1$; we here use the convexity of $\Omega$. Then $f^{\prime}(0)=0$ while $f^{\prime \prime}(\theta)=G_{i j}(t x+\theta z) z_{i} z_{j}>0$. Therefore $\phi(y)=\phi(t x+z)>\phi(t x)=t \phi(x)$. In this case (10) yields an inequality. This completes the proof of the lemma and, with it, that of the theorem.

3 . The case of concavity. We state below the theorem which is the companion of Theorem 1 for the case of concavity. Since its proof is the same as that of Theorem 1, we do not set it down.

THEOREM 2. Let $G(x)=c$ denote a concave family of surfaces. Let $\phi(x)$ be a positive function which satisfies the extremum property 


$$
\min _{y} \frac{\sum x_{i}^{*} y_{i}}{\phi(y)}=\frac{\sum x_{i}^{*} x_{i}}{\phi(x)}
$$

Then the solutions $\phi(x)$ of (12) are precisely given by the formula $\phi(x)$ $=k\left(\sum x_{i}^{*} x_{i}\right)^{1 / r}$ where $k$ is an arbitrary positive constant.

Furthermore, the inequality defined by (12) is strict; that is, for the value of $\phi(x)$ just given, and for a given $x$, the only solution $y$ of (12) is $y=t x$ where $t>0$.

4. The fundamental inequalities. We now proceed to derive inequalities in the classic form. It has been seen that we associate to any convex or concave surface two ray spaces, $\Omega$ and $\Omega^{*}$. There exists between the vectors of these spaces a one-to-one correspondence given by equation (2). Now in (10) and (12) our point of view so far has been that $x$ is arbitrary in $\Omega$ and $x^{*}$ is its correspondent. We now invert this point of view and choose $x^{*}$ first, thus making $x$ a function of $x^{*}$ given by (4). Thus we may write

$$
\frac{\sum x_{i}^{*} x_{i}}{\phi(x)}=\psi\left(x^{*}\right) \text {. }
$$

It is easy to see that $\psi\left(x^{*}\right)$ is homogeneous of degree 1 in $x^{*}$. Furthermore, if $\phi(x)=k\left(\sum x_{i}^{*} x_{i}\right)^{1 / r}$ then $\psi\left(x^{*}\right)=k^{-1}\left(\sum x_{i}^{*} x_{i}\right)^{1 / r^{\prime}}$, where $1 / r+1 / r^{\prime}=1$. In other words $\psi\left(x^{*}\right)$ is constant on the surface $\sum x_{i}{ }^{*} x_{i}=1$ considered as being in the $x^{*}$ space. In the case of convexity, we have from (10)

$$
\sum x_{i}^{*} y_{i} \leqq \psi\left(x^{*}\right) \phi(y) .
$$

This inequality holds for all $x^{*} \in \Omega^{*}$ and $y \in \Omega$. For a given $y$, the equality is valid along precisely one ray in $\Omega^{*}$. This ray is defined by the equivalent equations (2) and (4).

The case of concavity is essentially the same except that the inequality in (14) is reversed.

The function $\phi(x)$ in (10) has the three important properties: (1) $\phi(x)>0$; (2) $\phi(t x)=t \phi(x)$ for $t>0$; and (3) $\phi(x+y) \leqq \phi(x)+\phi(y)$. Only (3) needs attention. We have from (14) for an arbitrary $z^{*}, \sum z_{i}^{*}\left(x_{i}+y_{i}\right) \leqq \psi\left(z^{*}\right)[\phi(x)+\phi(y)]$. Now since for a particular $z^{*}$, namely for $z^{*}=(x+y)^{*}, \sum z_{i}{ }^{*}\left(x_{i}+y_{i}\right)=\psi\left(z^{*}\right)$ - $\phi(x+y)$, we obtain (3). It is clear that we have equality in (3) if and only if $x$ and $y$ are related by an expression $y=t x, t>0$.

5. Applications. The Hölder inequality. As has been mentioned earlier, the ease or difficulty of making applications of this theory is that of inverting a system of equations (2) to obtain (4) and that of determining whether a given quadratic form (3) is positive definite. Both of these questions are completely trivial in the basic inequalities of analysis which we treat here.

To obtain the Hölder inequality, consider the function $r G(x)=x_{1}^{r}+\cdots$ $+x_{n}^{r}$. Let $r>1$, and let $\Omega$ be the ray-space of all $x$ such that $x_{i}>0, i=1, \cdots, n$. In the first place $G(x)$ possesses all the derivatives we shall require. Next 
$x_{i}^{*}=x_{i}^{r-1}$ and $G_{i j}(x)=0$ if $i \neq j$, while $G_{i i}(x)=(r-1) x_{i}^{r-2}$. The matrix $\left\|G_{i j}(x)\right\|$ is a diagonal matrix in which the terms in the diagonal are positive; hence the associated quadratic form (3) is positive definite. Thus $G(x)=c$ is a convex surface.

Next the equations $x_{i}^{*}=x_{i}^{r-1}$ are easily solved, giving $x_{i}=x_{i}^{* r^{\prime}-1}$. Note that $\Omega^{*}$ consists of all $x^{*}$ such that $x_{i}^{*}>0, i=1, \cdots, n$. Clearly $\sum x_{i}^{*} x_{i}$ $=x_{1}^{* r^{\prime}}+\cdots+x_{n}^{* *^{\prime}}$ and hence (14) gives

$$
\sum x_{i}^{*} y_{i} \leqq\left(x_{1}^{*^{r^{\prime}}}+\cdots+x_{n}^{*^{r^{\prime}}}\right)^{1 / r^{\prime}}\left(y_{1}^{r}+\cdots+y_{n}^{r}\right)^{1 / r}
$$

which is Hölder's inequality. If some $x_{i}{ }^{*}$ or $y_{j}$ is zero or negative, the inequality is strengthened. Note that the Cauchy-Schwarz inequality, $r=2$, gives rise to the identity transformation $x_{i}^{*}=x_{i}$ and the identity matrix $\left\|G_{i j}(x)\right\|$ $=\left\|\delta_{i j}\right\|$. Note also that if $r<1$ we obtain the inequality (15) with a reversal of sign.

Let us now derive the inequality between arithmetic and geometric means. Let $-n G(x)=\left(x_{1} \cdots x_{n}\right)^{-1}$. Then $G(x)$ is differentiable and homogeneous of degree $r=-n$. Let $\Omega$ consist of all $x$ such that $x_{i}>0, i=1, \cdots, n$. We have $x_{i}^{*}=\left(n x_{i}\right)^{-1}\left(x_{1} \cdots x_{n}\right)^{-1}$. This system may be solved for $x_{i}$; in fact, $\left(x_{i} x_{i}^{*}\right)^{n+1}=n^{-1}\left(x_{1}^{*} \cdots x_{n}^{*}\right)$. It is clear that $\Omega^{*}$ consists of all $x^{*}$ such that $x_{i}^{*}>0, i=1, \cdots, n$. Also $\sum x_{i}^{*} x_{i}=\left(x_{1} \cdots x_{n}\right)^{-1}=[\phi(x)]^{-n}$ and also $\sum x_{i}^{*} x_{i}=\left[n^{n}\left(x_{1}^{*} \cdots x_{n}^{*}\right)\right]^{1 /(n+1)}=\left[\psi\left(x^{*}\right)\right]^{n /(n+1)}$. Thus if the quadratic form $(r-1) \sum G_{i j}(x) z_{i} z_{j}$ is positive we obtain the inequality

$$
\sum x_{i}^{*} y_{i} \geqq n\left(x_{1}^{*} \cdots x_{n}^{*}\right)^{1 / n}\left(y_{1} \cdots y_{n}\right)^{1 / n}
$$

which may be written $\left(u_{1}+\cdots+u_{n}\right) / n \geqq\left(u_{1} \cdots u_{n}\right)^{1 / n}$ where we have set $u_{i}=x_{i}{ }^{*} y_{i}$ and hence $u_{i}$ is an arbitrary positive number.

It remains to examine the quadratic form. We write $X^{-1}=n\left(x_{1} \cdots x_{n}\right)$. Then $\partial x_{i}{ }^{*} / \partial x_{j}=-\left(x_{i} x_{j}\right)^{-1} X$ if $i \neq j$ and $-2 x_{i}^{-2} X$ if $i=j$. Hence $(r-1)$ $\cdot \sum G_{i j}(x) z_{i} z_{j}=-(r-1) X\left[\sum\left(x_{i} x_{j}\right)^{-1} z_{i} z_{j}+\sum x_{i}^{-2} z_{i}^{2}\right]=(n+1) X\left[\left(\sum z_{i} / x_{i}\right)^{2}\right.$ $\left.+\sum\left(z_{i} / x_{i}\right)^{2}\right]>0$ for $z \neq 0$.

\section{Approximation by differentiable surfaces}

1. Problems under discussion. Up to the present moment we have considered one surface and its homothetic images. This surface gives rise to an adjoint surface and to two ray-spaces. In the present chapter we shall wish to consider simultaneously two or more distinct surfaces. We introduce the notion of distance between surfaces. In order to do this we shall have to confine ourselves to classes of surfaces which along with their adjoints are defined over the same pair of ray-spaces $\Omega$ and $\Omega^{*}$. Furthermore, the classes must be closed under a suitable operation which is a type of addition and gives rise to the addition of quadratic forms. Indeed the entire reason for introducing this operation is that the parallel operation on quadratic forms is so fertile. These classes of surfaces will be called natural classes. They are 
introduced in the next section.

Given a natural class of surfaces, we shall establish the following separation property: If $\mathfrak{S}_{0}$ and $\mathfrak{S}_{1}$ are such that $\mathfrak{S}_{0}$ separates $\mathfrak{S}_{1}$ from the origin, then for the adjoint surfaces, $\mathfrak{S}_{1}^{*}$ separates $\mathfrak{S}_{0}^{*}$ from the origin. With the help of this theorem, a useful metric may be introduced to measure the distance between two surfaces of a natural class. This distance has the property that its value is unchanged in passing to the adjoint. Thus it is possible to show that converging sequences of convex or concave surfaces do define limit surfaces. In this way the most general type of convexity or concavity is introduced by completing classes of differentiable surfaces.

The most important natural class is that in which $\Omega$ and $\Omega^{*}$ consist of the entire space. This gives rise to closed convex surfaces and hence to convex bodies. We shall show that in the process of completing this class we obtain all bodies convex in the classical sense. This is done by proving that any convex polyhedron may be approximated by surfaces convex in our sense. As a consequence it follows that any property of closed differentiable surfaces which is not disturbed by limiting processes holds also for convex bodies.

2. Natural classes of surfaces. We consider two families of surfaces given by the equations $G^{(0)}(x)=c$ and $G^{(1)}(x)=c$. Suppose that both are defined over the same ray-space $\Omega$. Suppose further that the homogeneity degree $r$ is the same for both functions. Consider the function $\lambda G^{(0)}(x)+\mu G^{(1)}(x)$ where $\lambda$ and $\mu$ are positive parameters; consider also the family of surfaces defined by setting this function equal to a constant. This operation defines an addition of families of surfaces. Note that the sum depends on $r$ as well as the geometrical properties of the initial surfaces. This new family generates a transformation (2) whose equations are $x_{i}{ }^{*}=\lambda G_{i}^{(0)}(x)+\mu G_{i}^{(1)}(x), i=1, \cdots, n$. The quadratic form associated with the transformation is $(r-1) \sum\left[\lambda G_{i j}^{(0)}(x)\right.$ $\left.+\mu G_{i j}^{(1)}(x)\right] z_{i} z_{j}$. Now, if the original families are both convex or both concave, then the same is true of their sum. This arises from the important fact that the sum of two positive definite quadratic forms enjoys the same property.

We introduce the notion of a natural class by considering the most important case. Suppose that we are concerned only with convexity. Suppose further that we are dealing with families whose domain of definition $\Omega$ is the entire $x$-space. For a given family, construct the set $\Omega^{*}$. Then since the rays in $\Omega$ and $\Omega^{*}$ are related by a one-to-one continuous correspondence, and since $\Omega^{*}$ is an open set, $\Omega^{*}$ must also consist of the entire space. Thus if a given convex surface is closed, so is its adjoint surface. Now if we consider two families of closed convex surfaces, then their sum certainly has the same property. In other words, the class of families for which $\Omega$ is the entire $x$-space and $\Omega^{*}$ is the entire $x^{*}$-space is closed under the operation of addition of families. This gives us the most important natural class. For the general situation we introduce the following

Definition 3. A class of families of surfaces is said to be a natural class 
providing that:

(a) There is a common domain $\Omega$ in which each family is defined; there is a common domain $\Omega^{*}$ in which each adjoint family is defined.

(b) The class is closed under the operation of positive scalar multiplication and addition; that is, if $G^{(0)}(x)=c$ and $G^{(1)}(x)=c$ belong to the class, so does $\lambda G^{(0)}(x)+\mu G^{(1)}(x)=c$ for $\lambda>0, \mu>0$.

(c) All the surfaces in the class are convex or all are concave.

Note that (b) implies that $\Omega^{*}$ is a convex set in the following sense: Given $x \in \Omega, x_{i}^{(0) *}=G_{i}^{(0)}(x), x_{i}^{(1) *}=G_{i}^{(1)}(x)$, then $\lambda x^{(0) *}+\mu x^{(1) *} \in \Omega^{*}$.

3. The separation property. Consider two surfaces $\mathfrak{S}_{0}$ and $\mathfrak{S}_{1}$. Let $\Im_{k}$ correspond to the equation $r G^{(k)}(x)=1, k=1,2$. Suppose that the functions $G^{k}(x)$ are defined over a common ray-space $\Omega$. We shall say that $\mathfrak{S}_{0}$ separates $\Im_{1}$ from the origin providing that every segment joining the origin to a point of $\mathfrak{S}_{1}$ intersects $\mathfrak{S}_{0}$. Thus the condition for separation of surfaces which are both convex or both concave is that

$$
r \sum\left[G_{i}^{(0)}(x)-G_{i}^{(1)}(x)\right] x_{i}>0
$$

for all $x \in \Omega$.

We shall now establish a theorem which allows us to introduce a metric into the class of convex and concave surfaces.

TheOREM 3. Let $\mathcal{C}$ be a natural class of families of surfaces. Consider two surfaces in $\mathcal{C}, \mathfrak{S}_{0}$ and $\mathfrak{S}_{1}$, which are defined by the equations $r G^{(k)}(x)=1$, $k=1,2$. Let the adjoint surfaces defined by the equations $r^{\prime} F^{(k)}(x)=1$ be denoted by $\mathfrak{\Im}_{0}^{*}$ and $\mathfrak{\Im}_{1}{ }^{*}$. Then $\mathfrak{\Im}_{0}$ separates $\mathfrak{\Im}_{1}$ from the origin if and only if $\mathfrak{\Im}_{1} *$ separates $\mathfrak{S}_{0} *$ from the origin.

We note first that the quadratic form $(r-1) \sum\left[t G_{i j}^{(0)}(x)+(1-t) G_{i j}^{(1)}(x)\right] z_{i} z_{j}$ is positive for any $t, 0 \leqq t \leqq 1$. Furthermore, since the given surfaces belong to a natural class, there is a common pair of ray-spaces $\Omega$ and $\Omega^{*}$ in which the operations to follow all take place.

Consider the surface $\mathfrak{S}_{t}$ defined by $\sum\left[t G_{i}^{(0)}(x)+(1-t) G_{i}^{(1)}(x)\right] x_{i}=1$. The transformation associated with it is

$$
x_{i}^{*}=t G_{i}^{(1)}(x)+(1-t) G_{i}^{(0)}(x) .
$$

Solving for $x_{i}$, we obtain

$$
x_{i}=H_{i}\left(t, x^{*}\right)
$$

where $H_{i}\left(0, x^{*}\right)=F_{i}^{(0)}\left(x^{*}\right)$ and $H_{i}\left(1, x^{*}\right)=F_{i}^{(1)}\left(x^{*}\right)$. Now

$$
r^{\prime} \sum H_{i}\left(t, x^{*}\right) x_{i}^{*}=r^{\prime} \sum\left[t G_{i}^{(1)}(x)+(1-t) G_{i}^{(0)}(x)\right] x_{i} .
$$

To prove the theorem we proceed as follows. Let $x^{*}$ be arbitrary but fixed. Then $x_{i}$ in (19) is a function of $t$. It will be shown that the derivative of (20) 
with respect to $t$ is positive for all $t, 0 \leqq t \leqq 1$. This will establish the fact that $r^{\prime} \sum\left[H_{i}\left(1, x^{*}\right)-H_{i}\left(0, x^{*}\right)\right] x_{i}^{*}>0$ and hence that $\mathfrak{S}_{1}{ }^{*}$ separates $\mathfrak{S}_{0}{ }^{*}$ from the origin. First we have

$$
\begin{aligned}
\frac{d}{d t} \sum H_{i}\left(t, x^{*}\right) x_{i}^{*}= & \sum\left[G_{i}^{(1)}(x)-G_{i}^{(0)}(x)\right] x_{i} \\
& +\sum\left[t G_{i j}^{(1)}(x)+(1-t) G_{i j}^{(0)}(x)\right] x_{i} \frac{d x_{j}}{d t} \\
& +\sum\left[t G_{i}^{(1)}(x)+(1-t) G_{i}^{(0)}(x)\right] \frac{d x_{i}}{d t}
\end{aligned}
$$

By Euler's theorem and symmetry properties, the last term in (21) equals $(r-1)^{-1} \sum\left[t G_{i j}^{(1)}(x)+(1-t) G_{i j}^{(0)}(x)\right] x_{i} d x_{j} / d t$. Also, differentiating (18) we have

$$
\begin{aligned}
0=G_{i}^{(1)}(x)-G_{i}^{(0)}(x)+\sum\left[t G_{i j}^{(1)}(x)+(1-t) G_{i j}^{(0)}(x)\right] & \frac{d x_{j}}{d t}, \\
& \\
i & =1, \cdots, n .
\end{aligned}
$$

If the equations (22) are multiplied by $x_{i}$ and summed with respect to $i$, and if the ensuing result is substituted in (21), the right side of (21) becomes $-(r-1)^{-1} \sum\left[G_{i}^{(1)}(x)-G_{i}^{(0)}(x)\right] x_{i}$. We now multiply this quantity by $r^{\prime}$ $=r(r-1)^{-1}$ and discover that it is positive by virtue of the hypothesis (17). This concludes the proof.

4. Introduction of the metric. Consider any two surfaces $\mathfrak{S}_{0}$ and $\mathfrak{S}_{1}$ which belong to the same natural class of families of surfaces. It may be that by a suitable magnification with the origin as center $\mathfrak{S}_{0}$ may be replaced by a surface similar to itself and such that $\mathfrak{S}_{1}$ separates it from the origin. In the same way, by a suitable contraction, $\mathfrak{S}_{0}$ may be replaced by a surface which separates $\mathfrak{S}_{1}$ from the origin. This will certainly be the case if the points of $\Im_{k}, k=1,2$, are bounded away from the origin and away from infinity. It may also be the case in other situations. We now suppose that this may be done for the surfaces $\Im_{k}$.

Assume that $\mathfrak{S}_{1}$ does not separate $\mathfrak{S}_{0}$ from the origin. Let $\alpha$ be the greatest lower bound of all numbers $m$ having the property that when $\widetilde{S}_{0}$ is magnified by a factor $m$, then $\widetilde{S}_{1}$ separates it from the origin. If $\mathfrak{S}_{1}$ does separate $\Im_{0}$ from the origin, set $\alpha=1$. Thus in any case $\alpha \geqq 1$. If $\Im_{0}$ does not separate $\Im_{1}$ from the origin, let $\beta$ be the least upper bound of all numbers $c$ such that if $\mathfrak{S}_{0}$ is contracted by the factor $c$ (note that $c<1$ ), then it separates $\mathfrak{S}_{1}$ from the origin. In case $\mathfrak{S}_{0}$ does separate $\mathfrak{S}_{1}$ from the origin, set $\beta=1$. Thus in any case, $0<\beta \leqq 1$.

It is clear that a suitable function of the numbers $\alpha$ and $\beta$ could probably serve as a distance from $\mathfrak{S}_{0}$ to $\mathfrak{S}_{1}$. If we apply the above procedure re- 
versing the role of the $\mathfrak{S}_{k}$ we obtain as our two numbers $\beta^{-1}$ and $\alpha^{-1}$. This prompts the following definition.

Definition 4. The number $\delta(0,1)=\log \alpha / \beta$ is said to be the distance from the surface $\mathfrak{\Im}_{0}$ to the surface $\mathfrak{S}_{1}$.

It is clear that (i) $\delta(0,1) \geqq 0 ; \delta(0,1)=0$ if and only if $\mathfrak{S}_{0}$ is identical with $\mathfrak{S}_{1}$; (ii) $\delta(0,1)=\delta(1,0)$; and (iii) if $\Im_{k}$ are three surfaces, $k=0,1,2$, then $\delta(0,2) \leqq \delta(0,1)+\delta(1,2)$. To prove (iii), note that if $\alpha_{i j}$ denotes the number $\alpha$ corresponding to $\mathfrak{S}_{i}$ and $\mathfrak{S}_{j}$, then $\alpha_{02} \leqq \alpha_{01} \cdot \alpha_{12}$. Similarly $\beta_{02} \geqq \beta_{01} \cdot \beta_{12}$; then (iii) follows from properties of the logarithmic function.

Now suppose a surface $\mathfrak{S}$ is magnified by a factor $m$. Then the conjugate surface is contracted by a factor $m^{-1}$. For if the equations of $\mathfrak{S}$ and $\mathfrak{S}^{*}$ are $G_{i}(x) x_{i}=\sum F_{i}\left(x^{*}\right) x_{i}{ }^{*}=1$, then after magnification, it is clear that $\subseteq$ has the equation $\sum G_{i}\left(m^{-1} x\right) m^{-1} x_{i}=1$ and from this follows that $\mathfrak{S}^{*}$ has the equation $\sum F_{i}\left(m x^{*}\right) m x_{i}^{*}=1$, which proves our statement.

Consider two surfaces $\mathfrak{S}_{0}$ and $\mathfrak{S}_{1}$ along with their adjoint surfaces $\mathfrak{S}_{0}{ }^{*}$ and $\mathfrak{S}_{1}^{*}$. Suppose that in magnifying $\mathfrak{S}_{0}$ by a factor $m$ it is transformed into a surface which is separated from the origin by $\Im_{1}$. Then by Theorem 3 and by the previous discussion, $\mathfrak{S}_{0}^{*}$ is contracted by the factor $m^{-1}$ and is transformed into a surface which separates $\widetilde{S}_{1}^{*}$ from the origin. Let us now compute the distance $\delta^{*}(0,1)=\log \alpha^{*} / \beta^{*}$ between $\mathfrak{S}_{0}^{*}$ and $\mathfrak{S}_{1}^{*}$. We see directly that $\alpha^{*}=\beta^{-1}$ and $\beta^{*}=\alpha^{-1}$. Hence $\log \alpha^{*} / \beta^{*}=\log \alpha / \beta$. Thus we see that the distance $\delta(0,1)$ between two surfaces $\mathfrak{S}_{0}$ and $\mathfrak{S}_{1}$ is identical with the distance $\delta^{*}(0,1)$ between their adjoint surfaces $\mathfrak{S}_{0}^{*}$ and $\mathfrak{S}_{1}^{*}$.

5. Completion of the space of surfaces. We have seen above that if $\mathcal{C}$ is a natural class of families of surfaces and if $\mathfrak{S}_{0}$ and $\mathfrak{S}_{1}$ are two surfaces in this class, then there may exist a real number $\delta(0,1)$ measuring the distance between the surfaces. In some classes this distance exists for every pair of surfaces. This is the case, for example, for the class of closed convex differentiable surfaces. It is clear that if for three surfaces $\Im_{k}, k=1,2,3$, the distances $\delta(0,1)$ and $\delta(1,2)$ exist, then so does $\delta(0,2)$. Thus the surfaces of a class may be partitioned into nonoverlapping subclasses having the property that any two surfaces $\Im_{k}, \Im_{l}$ of the same subclass give rise to a distance $\delta(k, l)$ whereas two surfaces of distinct classes give rise to no such function.

In a given subclass $\mathcal{D}$ of $\mathcal{C}$, consider any sequence $\left\{\Im_{k}\right\}$ which has the property that $\delta(k, l) \rightarrow 0$ as $k \rightarrow \infty$ and $l \rightarrow \infty$. Such a sequence is called a convergent sequence of surfaces. It is clear that since the surfaces in $\mathcal{D}$ constitute a metric space, we may construct a new metric space $\overline{\mathcal{D}}$ which is the completion of $\mathcal{D}$ by means of convergent sequences.

We shall show presently that if $\left\{\Im_{k}\right\}$ is a convergent sequence of surfaces in $\mathcal{D}$ then there exists a surface $\mathbb{S}$ to which the surfaces $\mathfrak{S}_{k}$ converge, $\mathfrak{S}_{k} \rightarrow \mathfrak{S}$. Furthermore, if $\Im_{k}^{*}$ is the adjoint of $\Im_{k}$, then $\left\{\Im_{k}^{*}\right\}$ is also a convergent sequence, since $\delta(i, j)=\delta^{*}(i, j)$. The surface to which $\left\{\Im_{k}^{*}\right\}$ converges will be denoted by $\mathfrak{S}^{*}, \mathfrak{S}_{k}^{*} \rightarrow \mathfrak{S}^{*}$. It may be seen that $\mathfrak{S}^{*}$ is independent of the 
particular sequence $\left\{\mathfrak{S}_{k}^{*}\right\}$. That is, if $\left\{\mathfrak{T}_{k}\right\}$ is a sequence equivalent to $\left\{\mathfrak{S}_{k}\right\}$, then $\left\{\mathfrak{T}_{k}^{*}\right\}$ is equivalent to $\left\{\widetilde{S}_{k}^{*}\right\}$. This process thus enlarges the class of pairs $\left(\mathfrak{S}, \mathfrak{S}^{*}\right)$ consisting of a surface and its adjoint.

Let $\left\{\Im_{k}\right\}$ be a convergent sequence. Consider any direction $\xi$ in $\Omega$ and let $\rho_{k}$ denote the length of the vector from the origin to the point on $\mathfrak{S}_{k}$ which lies in the direction $\xi$. It is easily seen that $\left|\log \rho_{k} / \rho_{l}\right| \leqq \delta(k, l)$. Thus the sequence $\left\{\log \rho_{k}\right\}$ is a convergent sequence. Suppose that $\rho_{k} \rightarrow \rho$. We define a surface $\subseteq$ to consist of the totality of points $\rho \xi$. It may be seen that the surface $\subseteq$ is continuous. Also, if the surfaces $\Im_{k}$ are closed and bound a convex body, then $\subseteq$ is closed and bounds a convex body. If the surfaces $\mathfrak{S}_{k}$ have the equations $\sum G_{i}^{(k)}(x) \cdot x_{i}=1$, then $\subseteq$ has the equation $S(x)=1$ where $S(x)$ $=\lim _{k \rightarrow \infty} \sum G_{i}^{(k)}(x) \cdot x_{i}$. Thus $S(x)$ is homogeneous of degree $r$. It is clear that, in general, $S(x)$ is not differentiable. Hence for $S(x)$, the transformation (4) and the quadratic form (5) are meaningless. We collect these results in a

TheOREM 4. Let $\mathcal{C}$ be a natural class and let $\mathcal{D}$ be a subclass of $\mathcal{C}$ such that the distance function for surfaces is defined for any two members of $\mathcal{D}$. If $\mathfrak{S}_{0}$ and $\Im_{1}$ are surfaces in $\mathcal{D}$, then $\delta(0,1)=\delta^{*}(0,1)$ where $\delta^{*}(0,1)$ represents the distance between the adjoint surfaces $\Im_{0}^{*}$ and $\Im_{1}^{*}$. A given sequence of surfaces $\left\{\Im_{k}\right\}$ converges if and only if the sequence of adjoints $\left\{\Im_{k}^{*}\right\}$ converges. If $\mathfrak{S}_{k} \rightarrow \mathfrak{S}$ and $\mathfrak{S}_{k}^{*} \rightarrow \mathfrak{S}^{*}$, then the mapping $\mathfrak{S}_{\leftrightarrow} \mathfrak{S}^{*}$ is a one-to-one isometric mapping of the closure of $\mathcal{D}$ onto the closure of $\mathcal{D}^{*}$, the class of surfaces adjoint to $\mathcal{D}$.

It should be noted that our original metric as given in Definition 4 may be applied to measure distances in $\overline{\mathcal{D}}$ and is identical with the metric constructed by considering convergent sequences in $\mathscr{D}$. $(\overline{\mathcal{D}}$ denotes the closure of $\mathscr{D}$.)

6. Extension of earlier inequalities. We have seen that for an arbitrary surface and its adjoint, we have an inequality of the type (14). Thus such an inequality is valid for every surface in the class $\mathcal{D}$ described above. Now we shall see that inequalities of this kind may be established for any surface in $\overline{\mathcal{D}}$, the closure of $\mathcal{D}$.

Let $\left\{\Im_{k}\right\}$ be a convergent sequence of surfaces in $\mathcal{D}$. Let $\Im_{k} \rightarrow \Im$ S. Suppose the equation of $\mathfrak{S}_{k}$ is $\phi_{(k)}(x)=1$ where $\phi_{(k)}(x)$ is homogeneous of degree 1 . It may be seen from the discussion in $\S 5$ that $\left\{\phi_{(k)}(x)\right\}$ is a convergent sequence, that $\phi_{(k)}(x) \rightarrow \phi(x)$, and that $\phi(x)=1$ is the equation of $\mathfrak{S}$. In the same way, if $\Im_{k}^{*} \rightarrow \Im^{*}$ and if $\psi_{(k)}\left(x^{*}\right)=1$ is the equation of $\Im_{k^{*}}^{*}$, then $\psi_{(k)}\left(x^{*}\right) \rightarrow \psi\left(x^{*}\right)$ where $\psi\left(x^{*}\right)=1$ is the equation of $\mathfrak{S}^{*}$.

Now if we assume for the moment that we are treating the convex case, with either $\Omega$ or $\Omega^{*}$ a convex set, we have for every $k$ and for all vectors $x^{*}, y: \sum x_{i}^{*} y_{i} \leqq \psi_{(k)}\left(x^{*}\right) \phi_{(k)}(y)$. Hold $x^{*}$ and $y$ fixed and consider the limit as $k \rightarrow \infty$. We obtain

$$
\sum x_{i}^{*} y_{i} \leqq \psi\left(x^{*}\right) \phi(y) .
$$

The inequality holds for all $x^{*} \in \Omega^{*}$ and $y \in \Omega$. 
The cited work of Fenchel gives the broadest conditions under which this inequality (in an altered form) is satisfied.

7. Application to convex bodies. In this section we prove that a surface which bounds a convex body may be approximated by differentiable surfaces which are convex in our sense. It will be sufficient to show that any convex polyhedron may be so approximated, for the latter lie dense in the sense of our metric in the space of convex bodies. Specifically, we shall prove

THEOREM 5. Let $\mathfrak{B}$ be a polyhedron with the origin as interior point and convex in the classical sense. Then it is possible to approximate to $\mathfrak{B}$ arbitrarily closely in terms of the metric of Definition 4 by analytic surfaces which are convex in our sense.

Proof. Let $\mathfrak{B}$ be determined by $s$ hyperplanes whose equations are given by

$$
L_{i}+1=\sum a_{i j} x_{i}+1=0, \quad i=1, \cdots, s .
$$

Then the points $x$ for which $L_{i}+1 \geqq 0$ for each $i$ constitute $\mathfrak{B}$ and its interior.

Let $\epsilon$ be a positive number less than one, $0<\epsilon<1$. Consider the algebraic surface

$$
S(x) \equiv \prod\left(L_{i}+1\right)=\epsilon
$$

where the product index runs from $i=1$ to $i=s$. The origin is not on this surface. If $\sigma$ represents a line segment joining the origin to an arbitrary point of $\mathfrak{B}$, then $\sigma$ intersects the surface at precisely one point. The totality of these points constitutes a surface $\mathfrak{S}_{\epsilon}$. This surface may be better described as follows: Let $\xi$ be a direction and let $x=\xi t,-\infty<t<\infty$, represent the line through the origin in the direction $\xi$. The polynomial equation $S(\xi t)=0$ is of degree $s$ in $t$ unless $\xi$ is parallel to some hyperplane in (24), and all of its roots are real. Thus $S(\xi t)=\epsilon$ has precisely one positive root $t_{1}$ such that $t_{1} \xi$ lies inside $\mathfrak{B}$. This root is the smallest positive root of the polynomial. The totality of points $x=t_{1} \xi$ constitute $\Im_{\epsilon}$. It is clear that $\widetilde{S}_{\epsilon}$ is an analytic surface. It is also clear that if $\left\{\epsilon_{n}\right\}$ is a sequence for which $\epsilon_{n} \rightarrow 0$, then $\left\{\Im_{\epsilon_{n}}\right\}$ is a convergent sequence of surfaces converging to $\mathfrak{B}$. It remains to show that $\mathfrak{S}_{\epsilon}$ is convex.

Our first task is to obtain an expression for $S(x)=\epsilon$ which is homogeneous. We write $c x$ for $x$, obtaining $S(c x)=\epsilon$. Solving this for $c$ we obtain a function homogeneous of degree $-1, c=H(x)$. Thus we have

$$
S(c x)=\epsilon \text { or } H(x)=c .
$$

In order to deal effectively with some computational difficulties, we introduce

Lemma 4. Let $H(x)$ be a positive-valued function which is positively homogeneous of degree -1 . Let $r>1$ and let $K(x)=H^{-r}(x)$. Then in order that $\sum K_{i j}(x) z_{i} z_{j}$ be a positive definite quadratic form it is sufficient that $\sum H_{i j}(x) z_{i} z_{j}$ 
be negative definite for those vectors $z$ which lie in the tangent hyperplane at $x: \sum H_{i}(x) z_{i}=0$.

Note that $K(x)$ is homogeneous of degree $r>1$. We have $K_{i j}$ $=r(r+1) H^{-(r+2)} H_{i} H_{j}-r H^{-(r+1)} H_{i j}$. Thus $\sum K_{i j} z_{i} z_{j}$ is the sum of two terms: the first is $r(r+1) H^{-(r+2)}\left(\sum H_{i} z_{i}\right)^{2}$ which equals zero in the tangent hyperplane; the second is $-r H^{-(r+1)} \sum H_{i j} z_{i} z_{j}$ which is positive in the tangent hyperplane by hypothesis. Thus $\sum K_{i j} z_{i} z_{j}$ is positive in the tangent hyperplane if $z \neq 0$.

For an arbitrary $z$, write $z=t x+y$ where $y$ lies in the tangent hyperplane. Then $\sum K_{i j} z_{i} z_{j}=\sum K_{i j} y_{i} y_{j}+2 t \sum K_{i j} x_{i} y_{j}+t^{2} \sum K_{i j} x_{i} x_{j}$. Since $\sum K_{i j} x_{i} y_{j}=0$ and $\sum K_{i j} x_{i} x_{j}=r(r-1) K(x)$, the lemma is proved.

To prove Theorem 5 , it is sufficient to show that $\sum H_{i j} z_{i} z_{j}<0$ for all $z$ which satisfy $\sum H_{i} z_{i}=0, z \neq 0$.

We write $c_{i}$ for $\partial c / \partial x_{i}$ (which is the same as $\partial H / \partial x_{i}$ ); also, $S_{j}$ denotes the derivative of $S$ with respect to the $j$ th argument: $c x_{j}$. Thus we obtain $\left(\sum S_{k} x_{k}\right) c_{i}+c S_{i}=0$. If this be differentiated once more, we have

$$
\begin{aligned}
\left(\sum S_{k} x_{k}\right) c_{i j}+\left(\sum S_{k l} x_{k} x_{l}\right) c_{i} c_{j}+c \sum S_{k j} x_{k} c_{i}+S_{j} c_{i}+S_{i} c_{j} & +c^{2} S_{i j} \\
& +c\left(\sum S_{i k} x_{k}\right) c_{j}=0 .
\end{aligned}
$$

Now consider the expression $\sum c_{i j} z_{i} z_{j}$ where $z$ lies in the tangent hyperplane of $S(c x)=\epsilon$. Then $\sum_{i} S_{i} z_{i}=0$ and hence $\sum c_{i} z_{i}=0$. Thus

$$
\left(\sum S_{k} x_{k}\right) \sum c_{i j} z_{i} z_{j}=-c^{2} \sum S_{i j} z_{i} z_{j} \text {. }
$$

At this point it is convenient to consider that the equation of the surface $\widetilde{S}_{\epsilon}$ is not given in the form (25) but in the equivalent form $S(x)$ $=\sum \log \left(L_{k}+1\right)-\log \epsilon=0$. We have $\sum S_{i j} z_{i} z_{j}=-\sum\left(a_{k i} a_{k j} z_{i} z_{j}\right)\left(L_{k}+1\right)^{-2}$ $=-\sum L_{\boldsymbol{k}}^{2}(z)\left(L_{k}(x)+1\right)^{-2}$. Thus $\sum S_{i j} z_{i} z_{j} \leqq 0$ for all $z$. If this quantity equals zero, then $L_{k}(z)=0$ for each $k$. In turn, this means that $z$ is parallel to each hyperplane defining $\mathfrak{P}$. Since $\mathfrak{B}$ is a closed surface, this cannot arise. We see therefore that $-c^{2} \sum S_{i j} z_{i} z_{j}>0$ if $z \neq 0$.

We now examine the quantity $\sum S_{k} x_{k}=\sum L_{k}\left(L_{k}+1\right)^{-1}$ which appears in (27). First, $L_{k}(x)+1>0$ inside $\mathfrak{B}$. Also, if the point $x$ is close to the hyperplane $L_{j}(x)+1=0$, then $L_{j}\left(L_{j}+1\right)^{-1}$ is negative and large in absolute value. Thus if $x$ is sufficiently close to $\$$-in other words, if $\epsilon$ is sufficiently smallthen $\sum S_{k} x_{k}<0$. Now the locus $\sum S_{k} x_{k}=0$ is a closed set. Thus it is easy to see that there is a number $\epsilon_{0}$ such that if $\epsilon<\epsilon_{0}$, then $\sum S_{k} x_{k}<0$ for all $x$ on $\Im_{\text {e. }}$ Hence $\sum c_{i j} z_{i} z_{j}<0$ for all $z$ in the tangent hyperplane and the theorem follows from Lemma 4.

\section{THE THEORY OF VOLUMES AND MIXED VOLUMES}

1. Scope of chapter. In this chapter we consider the natural class of closed convex differentiable surfaces. Such a surface $\subseteq$ encloses a volume $V$. 
Formulae are developed for the volume of $V$ (or rather of $V^{*}$, since that is more convenient) making use of the fundamental transformation (2). The volume is then expressed by means of a surface integral over the unit sphere. This leads to a third expression for the volume (see equation (33)) which is fundamental to the theory. A certain positive definite quadratic form leads to the introduction of the notion of partial volume.

The theory of mixed volumes due to Minkowski may then be developed. The addition of convex bodies is significant precisely because it has as its algebraic counterpart the addition of positive definite quadratic forms. The elementary properties of mixed volumes are easily derived from earlier formulae. It is shown how each such mixed volume may be represented as the trace of a matrix. Finally certain fundamental formulae and functions are introduced and studied.

2. A formula for volume. Consider the convex surface $r G(x)=1, r>1$. Here the domain of definition $\Omega$ is the entire $x$-space. Its adjoint surface is $r^{\prime} F\left(x^{*}\right)=1$ and $\Omega^{*}$ is the entire $x^{*}$-space. Let the volume enclosed by the latter be denoted by $V^{*}$. Then $V^{*}=\int d V^{*}$ where the integral sign denotes an $n$-fold integration in $x^{*}$-space over $V^{*}$. Now consider the transformation $x_{i}{ }^{*}=G_{i}(x)$ given in (2) and its inverse $x_{i}=F_{i}\left(x^{*}\right)$. This is a one-to-one transformation of $\Omega$ onto $\Omega^{*}$ which has a positive Jacobian. If we transform $\int d V^{*}$ in accordance with this transformation we obtain

$$
V^{*}=\int_{V^{*}} d V^{*}=\int_{V}\left|G_{i j}(x)\right| d V .
$$

This is the first formula for $V$.

3. A second formula. We show how $V^{*}$ may be expressed as an integral over the unit sphere. Let $\Omega$ represent the unit sphere in $n$ dimensions and let $d \omega$ represent an infinitesimal element of surface of $\Omega$. The present use of the symbol " $\Omega$ " and the use of chapters I, II do not conflict. For an arbitrary vector $x=\left(x_{1}, \cdots, x_{n}\right)$ let $|x|$ represent $\left(\sum x_{i}^{2}\right)^{1 / 2}$.

Suppose now that $x$ is a vector on the surface which bounds $V$. Then $c x$, $0 \leqq c \leqq 1$, represents a segment from the origin to $x$. Consider the conical volume bounded by $V$ and by the rays from the origin through the boundary of $d \omega$. We shall compute the integral (28) over this volume. Since $G_{i j}(x)$ is homogeneous of degree $r-2$, the determinant $\left|G_{i j}(x)\right|$ is homogeneous of degree $n(r-2)$. Thus for the given integral we have

$$
d \omega \int_{0}^{1}\left|G_{i j}(c x)\right| c^{n-1}|x|^{n} d c=[n(r-1)]^{-1}\left|G_{i j}(x)\right| \cdot|x|^{n} d \omega
$$

since $d|c x|^{n}=|x|^{n} n c^{n-1} d c$. Thus

$$
V^{*}=\frac{1}{n(r-1)} \int_{\Omega}\left|G_{i j}(x)\right| \cdot\left|x_{0}\right|^{n} d \omega
$$


where the variable $x$ is to be evaluated on the surface $r G(x)=1$ which bounds $V$ and not on $\Omega$.

The expression (29) is valid for all values of $r>1$ which define the homogeneity degree of the function $G(x)$. We recall that the surface bounding $V$ is given by an equation of the form $\phi(x)=1$ and then $r G(x)=\phi^{r}(x), r>1$. Thus in (29) we obtain a valid expression for $V^{*}$ if we take the limit of the integral as $r \rightarrow 1$. The integrand $\left|G_{i j}(x)\right| \cdot|x|^{n}$ is homogeneous of degree $n r-n$. Thus if $r$ is close to 1 , this degree is close to 0 , and the variable $x$ may be evaluated without great error over the sphere rather than on the surface $\phi(x)=1$. This leads then to the formula

$$
V^{*}=\lim _{r \rightarrow 1} \frac{1}{n(r-1)} \int_{\Omega}\left|G_{i j}(x)\right| d \omega .
$$

4. An important matrix. Consider the matrix $\left\|\phi_{i j}(x)\right\|$. We have seen before that it is singular. Let $\Phi_{i j}(x)$ represent the cofactor in this matrix of the element $\phi_{i j}(x)$. Thus $\Phi_{i j}(x)$ is a certain signed determinant with $n-1$ rows. Consider the matrix $\left\|\Phi_{i j}(x)\right\|$. We see that the latter is symmetric and that $\left\|\Phi_{i j}\right\| \cdot\left\|\phi_{i j}\right\|$ equals the zero matrix. We shall see in a moment that $\left\|\Phi_{i j}\right\|$ is not identically zero, that is, that $\left\|\phi_{i j}\right\|$ is of rank $n-1$.

Consider the function

$$
W(r)=\frac{1}{n} \int_{\Omega}\left|G_{i j}(x)\right| d \omega .
$$

It is clear that $W(1)=0$. Furthermore, formula (30) states that $V^{*}$ $=d W /\left.d r\right|_{r=1}$. We proceed to compute this derivative. Since $G_{i j}$ $=(r-1) \phi^{r-2} \phi_{i} \phi_{j}+\phi^{r-1} \phi_{i j}$, then $d G_{i j} / d r=\phi^{r-2} \phi_{i} \phi_{j}+(r-1) \phi^{r-2} \phi_{i} \phi_{j} \log \phi$ $+\phi^{r-1} \phi_{i j} \log \phi$. Hence

$$
\left.\frac{d}{d r} G_{i j}\right|_{r=1}=\phi^{-1} \phi_{i} \phi_{j}+\phi_{i j} \log \phi .
$$

By the rule for differentiating determinants we see that

$$
V^{*}=\left.\frac{1}{n} \int_{\Omega} \frac{d}{d r}\right|_{r=1}\left|G_{i j}\right| d \omega=\frac{1}{n} \int_{\Omega} \phi^{-1} \sum \Phi_{i j} \phi_{i} \phi_{j} d \omega .
$$

This expresses $V^{*}$ as the integral of a quadratic form. Since $V^{*} \neq 0$, the matrix $\left\|\Phi_{i j}\right\|$ is not zero.

We see that in the hyperplane tangent to $\Omega$ at the point $x,\left\|\phi_{i j}\right\|$ is nonsingular-note that the matrix is reduced by this hyperplane. It follows that $\left\|\Phi_{i j}\right\|$ is also reduced by this hyperplane and in it is the zero matrix. Thus the characteristic roots of $\left\|\Phi_{i j}\right\|$ are all zero except one. This one root obviously has the value $\alpha=\sum \Phi_{k k}$, the trace of $\left\|\Phi_{i j}\right\|$.

The components $\phi_{i}$ of the vector $\left(\phi_{1}, \cdots, \phi_{n}\right)$ may be resolved as fol- 
lows: $\phi_{i}=\lambda x_{i}+y_{i}$ where $\sum x_{i} y_{i}=0$ and where $\lambda$ is some constant. Thus $\sum \Phi_{i j} \phi_{j}=\sum \Phi_{i j}\left(\lambda x_{j}+y_{j}\right)=\alpha \lambda x_{i}$. Also $\phi=\sum x_{i} \phi_{i}=\lambda|x|^{2}$. Thus $\lambda=\phi|x|^{-2}$. Hence we have $\sum \Phi_{i j} \phi_{i} \phi_{j}=\sum \alpha \lambda x_{i} \phi_{i}=\phi^{2}|x|^{-2} \sum \Phi_{k k}$. Since $|x|=1$ on $\Omega$, we drop the term $|x|^{-2}$ henceforth. This gives the final and most sophisticated expression for $V^{*}$ :

$$
V^{*}=\frac{1}{n} \int_{\Omega} \phi\left[\Phi_{11}+\cdots+\Phi_{n n}\right] d \omega .
$$

It may be said in passing that several alternative derivations of (33) are possible.

5. A second matrix. Consider the matrix

$$
\left\|\frac{1}{n} \int_{\mathbf{\Omega}} \phi \Phi_{i j} d \omega\right\|
$$

whose element in the place $(i, j)$ is the indicated integral. It is clear that the trace of this matrix is precisely $V^{*}$. Since $\left\|\Phi_{i_{j}}\right\|$ is a non-negative matrix, its integral is likewise non-negative. We shall prove that the matrix (34) is in fact positive definite. Let $z$ be an arbitrary vector on the unit sphere. Then for an arbitrary $x, \sum \phi \Phi_{i j}(x) z_{i} z_{j} \geqq 0$. For $x=z$, this quantity is actually positive. Hence the matrix (34) is positive definite.

6. Partial volumes. We introduce the notion of partial volumes: $V^{*}(i, j)$, $i, j=1, \cdots, n$. Let $x$ be a fixed point on $\Omega$, let $z$ be an arbitrary vector, and write $z=\mu x+y$ where $\sum x_{i} y_{i}=0$. Then we have

$$
\sum \Phi_{i j} z_{i} z_{j}=\left(\sum \Phi_{k k}\right) \mu^{2}=\left(\sum \Phi_{k k}\right)\left(\sum x_{i} z_{i}\right)^{2} .
$$

Thus

$$
\Phi_{i j}=\left(\sum \Phi_{k k}\right) x_{i} x_{j}
$$

Now $n^{-1} \int_{\Omega} \sum \phi \Phi_{i j} x_{i} x_{j} d \omega=n^{-1} \int_{\Omega} \phi\left(\sum \Phi_{k k}\right)\left(\sum x_{i}^{2} x_{j}^{2}\right) d \omega=n^{-1} \int_{\Omega} \phi\left(\sum \Phi_{k k}\right) d \omega=V^{*}$. Let

$$
V^{*}(i, j)=\frac{1}{n} \int_{\Omega} \phi \Phi_{i j} x_{i} x_{j} d \omega .
$$

Then clearly, $\sum V^{*}(i j)=V^{*}$. This justifies the term "partial volume." The properties of these partial volumes are as yet unknown.

7. The addition of convex bodies. Up to the present point we have not encountered the concept of the addition of convex bodies. In this section we propose to show how this notion can be handled by the present theory. Let $\Omega_{0}$ and $\Omega_{1}$ be two convex bodies. Then if $x_{0}$ and $x_{1}$ are arbitrary vectors terminating in $\Omega_{0}$ and $\Omega_{1}$, consider the vector $x=x_{0}+x_{1}$. The totality of vectors $x$ constitutes a new convex body $\Omega$ for which we write $\Omega=\Omega_{0}+\Omega_{1}$. This is the classic definition of addition. 
Now let $\widetilde{S}_{0}$ and $\widetilde{S}_{1}$ be closed differentiable surfaces defined by $\phi_{(0)}(x)=1$ and $\phi_{(1)}(x)=1$; or alternatively by $r G^{(i)}(x)=\phi_{(i)}^{r}(x)=1$. Let $\Im_{i}^{*}$ be the conjugate surfaces. Consider now the surface $\mathfrak{S}=\mathfrak{S}(r)$ defined by $\phi_{(0)}^{r}(x)+\phi_{(1)}^{\tau}(x)=1$ or alternatively by $r\left(G^{(0)}(x)+G^{(1)}(x)\right)=1$. Clearly, it is convex. The adjoint surface $\mathfrak{S}^{*}=\mathfrak{S}^{*}(r)$ is then said to be formed from the surfaces $\mathfrak{S}_{0}^{*}$ and $\mathfrak{S}_{1}^{*}$ by addition of order $r$.

The adjoint of $\mathfrak{S}^{*}$, namely $\mathfrak{S}$, may be defined by the equation $\xi(x, r)$ $=\left[\phi_{(0)}^{r}(x)+\phi_{(1)}^{r}(x)\right]^{1 / r}=1$. Thus $\xi(x, r)$ is homogeneous of degree 1. Clearly, $\lim _{r \rightarrow 1} \xi(x, r)=\phi_{(0)}(x)+\phi_{(1)}(x)$. Thus the support function of $\mathfrak{S}^{*}(r)$ has as its limit as $r \rightarrow 1$, the sum of the support functions of $\mathfrak{S}_{0}^{*}$ and $\mathfrak{S}_{1}^{*}$. This means in turn that $\lim _{r \rightarrow 1} \mathfrak{S}^{*}(r)$ is the sum in the classic sense of $\mathfrak{S}_{0}^{*}$ and $\mathfrak{S}_{1}^{*}$. It may be seen that the above arguments may be extended to cover the addition of any finite number of surfaces.

8. Mixed volumes. We restrict ourselves to the case of two bodies $\mathfrak{S}_{0}$ and $\mathfrak{S}_{1}$. For the sake of avoiding a cumbersome notation, we assume that $\mathfrak{S}_{0}$ is defined by $r G(x)=1$ and $\Im_{1}$ by $r H(x)=1$. Let $\lambda$ and $\mu$ be arbitrary but fixed non-negative numbers. Then $\lambda S_{0}$ and $\mu \Im_{1}$ have an obvious meaning. The sum $\subseteq(r)$ of $\lambda \Im_{0}$ and $\mu \Im_{1}$ is defined by $r(\lambda G(x)+\mu H(x))=1$. The adjoint $\varsigma^{*}(r)$ has a volume $V^{*}(r)$ for which the formulas (28) and (29) apply directly but not (30), (32), or (33). For $\lim _{r \rightarrow 1} V^{*}(r)$, write $V^{*}$. Then from (29)

$$
V^{*}=\lim _{r \rightarrow 1} \frac{1}{n(r-1)} \int_{\Omega}\left|\lambda G_{i j}(x)+\mu H_{i j}(x)\right| \cdot|x|^{n} d \omega .
$$

The presumption is that $x$ in the integral should be evaluated on the surface of $\lim _{r \rightarrow 1} \mathfrak{S}(r)$. However, as $r \rightarrow 1$, the integrand becomes homogeneous of degree zero, hence we may evaluate $x$ over the surface of the unit sphere. This means that equations (30), (32), and (33) may be applied to the present problem and yield not $V^{*}(r)$ but simply $V^{*}$. In particular (30) becomes

$$
V^{*}=\lim _{r \rightarrow 1} \frac{1}{n(r-1)} \int_{\Omega}\left|\lambda G_{i j}(x)+\mu H_{i j}(x)\right| d \omega .
$$

The expression (37) may also be expressed as the derivative with respect to $r$ at $r=1$ of the given integral. It is clear that the integrand is a polynomial in the parameters $\lambda$ and $\mu$, homogeneous of degree $n$. Hence the expression for $V^{*}$ is also such a polynomial. For each $x$ the coefficient of $\lambda^{\nu} \mu^{n-\nu}$ in the polynomial $\left|\lambda G_{i j}+\mu H_{i j}\right|$ is a positive quantity. This follows from the elementary theory of positive definite symmetric matrices. Thus the integral of the coefficient is likewise positive. Finally the derivative with respect to $r$ at $r=1$ yields a coefficient which is not negative. As is usual, we write

$$
V^{*}=\sum C_{n, \nu} V_{\nu}^{*} \lambda^{n-\nu} \mu^{\nu} \text {. }
$$

The numbers $V_{\nu}^{*}$ are called the mixed volumes of the given sum. Thus for 
each $\nu, V_{\nu}^{*} \geqq 0$.

A fundamental problem for mixed volumes may be described as follows. By what we have stated above, the polynomial $\left|\lambda G_{i j}(x)+\mu H_{i j}(x)\right|$ has $n$ real roots which are negative. Thus its derivative at $r=1$ has $n$ real roots which are nonpositive. It is surmised that the integral of the latter polynomial over $\Omega$ has the same properties. Thus a property which is easily established in the small is surmised in the large. If this surmise is correct, a host of inequalities involving mixed volumes follows of which the Brunn-Minkowski theorem is but a special example.

9. The representation of mixed volumes as traces. We shall show that the mixed volume $V_{\nu}^{*}$ may be expressed in terms of the integral of the trace of a certain matrix. Since the integral of a trace equals the trace of the integral we have the result that $V_{\nu}^{*}$ may be expressed in terms of the trace of a matrix. The matrix for $V_{\nu}^{*}$ has $C_{n, \nu}$ rows and columns. In order to obtain this representation we consider first the concept of compound matrices.

Let $\left\|a_{i j}\right\|$ be a nonsingular matrix; in the present context, we are interested in the symmetric case. For a given integer $\nu, 1 \leqq \nu \leqq n$, we construct a matrix $\left\|a_{i j}\right\|^{(\nu)}$ whose elements are the $\nu$-rowed minors of $\left\|a_{i j}\right\|$. Thus $\left\|a_{i j}\right\|(v)$ has $C_{n, \nu}$ rows and columns. It is called the $\nu$ th compound matrix of $\left\|a_{i j}\right\|$. The rows may be arranged in some order, say the lexicographic order. This determines the order for the columns. Since $\left\|a_{i j}\right\|$ is symmetric, so are its compound matrices. It may also be seen that the mapping $\left\|a_{i j}\right\| \rightarrow\left\|a_{i j}\right\|(\boldsymbol{p})$ is a representation. That is, if for three matrices we have $\left\|a_{i j}\right\| \cdot\left\|b_{i j}\right\|=\left\|c_{i j}\right\|$, then we also have $\left\|a_{i j}\right\|^{(v)}\left\|b_{i j}\right\|^{(v)}=\left\|c_{i j}\right\|^{(v)}$. The proof of this rests on the following fact: If $x^{(1)}, \cdots, x^{(\nu)}$ are vectors which are simultaneously transformed by $\left\|a_{i j}\right\|$ into the vectors $y^{(1)}, \cdots, y^{(v)}$, then the vector whose components are the $\nu$-rowed determinants of the matrix $\left\|x_{j}^{(i)}\right\|, i=1, \cdots, \nu$; $j=1, \cdots, n$, are transformed into a similar vector formed from the matrix $\left\|y_{j}^{(i)}\right\|$ by the matrix $\left\|a_{i j}\right\|^{(v)}$. Use is made here of an extension of the theorem on the multiplication of determinants.

It may be seen that the $\nu$ th compound matrix of the identity matrix is itself the identity matrix -in a space of another dimension. Thus, if we have $\left\|a_{i j}\right\| \cdot\left\|b_{i j}\right\|=I$, then $\left\|a_{i j}\right\|^{(\nu)} \cdot\left\|b_{i j}\right\|(v)=I$. That is, the $\nu$ th compound of the inverse equals the inverse of the $\nu$ th compound.

Now let $A^{(v)}$ represent a matrix whose elements are the cofactors formed from $\left\|a_{i j}\right\|$ of the elements in $\left\|a_{i j}\right\|^{(v)}$. Then by the Laplace development, we have $\left.\left\|a_{i j}\right\|\right|^{(v)} \cdot A^{(v)}=\left|a_{i j}\right| \cdot I$, where $\left|a_{i j}\right|$ represents the determinant of $\left\|a_{i j}\right\|$. Thus $\left|a_{i j}\right|^{-1} A^{(v)}$ is the inverse of $\left\|a_{i j}\right\|^{(\nu)}$ and hence it is the $\nu$ th compound matrix of the inverse of $\left\|a_{i j}\right\|$.

Now let us return to mixed volumes. Consider the situation described in $\$ 8$ leading to equations (37) and (38). Let us examine $n V_{1}^{*}$, the coefficient of $\lambda^{n-1} \mu$. The coefficient of $\lambda^{n-1} \mu$ in the integrand (37) is a sum of $n$ determinants containing each one column of $\left|H_{i j}\right|$ and $n-1$ columns of $\left|G_{i j}\right|$. In other 
words, this coefficient equals $\sum H_{i j} G_{i j}$ where $G_{i j}$ is the cofactor of $G_{i j}$ in $\left|G_{i j}\right|$. Now $\left\|G_{i j}\right\| \cdot\left\|G_{i j}||=\left|G_{i j}\right| \cdot\right\| \delta_{i j}||$. Hence the coefficient of $\lambda^{n-1} \mu$ under the integral equals the trace of the matrix product $\left\|H_{i j}\right\| \cdot\left\|G_{i j}\right\|^{-1}$ multiplied by $\left|G_{i j}\right|$. If we indicate the trace of a matrix $A$ by $T(A)$ we may write

$$
n V_{1}^{*}=\lim _{r \rightarrow 1} \frac{1}{n(r-1)} \int_{\Omega}\left|G_{i j}(x)\right| T\left(\left\|H_{i j}(x)\right\| \cdot\left\|G_{i j}(x)\right\|^{-1}\right) d \omega .
$$

In a similar way, we obtain an expression for $C_{n, \nu} V_{\nu}^{*}$. The coefficient of $\lambda^{n-\nu} \mu^{\nu}$ in the integrand (37) is a sum of determinants containing each $\nu$ columns of $\left|H_{i j}\right|$ and $n-\nu$ columns of $\left|G_{i j}\right|$. It may be seen from the discussion which has preceded that this coefficient equals the trace of the matrix $\left(\left\|H_{i j}\right\| \cdot\left\|G_{i j}\right\|^{-1}\right)^{(v)}$ multiplied by $\left|G_{i j}\right|$. Thus we have

$$
C_{n, \nu} V_{\nu}^{*}=\lim _{r \rightarrow 1} \frac{1}{n(r-1)} \int_{\Omega}\left|G_{i j}(x)\right| T\left(\left\|H_{i j}(x)\right\| \cdot\left\|G_{i j}(x)\right\|^{-1}\right)^{(v)} d \omega .
$$

The formula is valid for all $\nu, 1 \leqq \nu \leqq n-1$. By a suitable definition of a compound matrix, we may consider (40) to apply also to the cases $\nu=0, n$ given in equation (30).

Consider the matrix $\left|G_{i j}(x)\right|\left(\left\|H_{i j}(x)\right\| \cdot\left\|G_{i j}(x)\right\|^{-1}\right)^{(v)}$. If we integrate its elements over the unit sphere, we obtain a matrix $\mathfrak{M}(G, H, \nu, r)$. Thus we have

$$
C_{n, \nu} V_{\nu}^{*}=\lim _{r \rightarrow 1} \frac{1}{n(r-1)} T[\mathfrak{M}(G, H, \nu, r)] .
$$

10. On the function $W(r)$. The function $W(r)$ was defined in equation (31). Since this function is the basis of the entire theory of volumes it is of importance to examine it a little more closely. We set down some equivalent expressions for it. First, since $G(x)=r^{-1} \phi^{r}(x), G_{i j}=(r-1) \phi^{r-2} \phi_{i} \phi_{j}+\phi^{r-1} \phi_{i j}$. Hence $\left|G_{i j}\right|=(r-1) \phi^{n(r-1)-1} \sum \Phi_{i j} \phi_{i} \phi_{j}$. Thus

$$
W(r)=\frac{(r-1)}{n} \int_{\Omega} \phi^{n(r-1)-1} \sum \Phi_{i j} \phi_{i} \phi_{j} d \omega .
$$

Let $\left|G_{i} G_{i 2} \cdots G_{i n}\right|$ represent the determinant whose $i$ th row contains the elements $G_{i}, G_{i 2}, \cdots, G_{i n}$. Then $(r-1)\left|G_{i} G_{i 2} \cdots G_{i n}\right|=\mid G_{i 1} x_{1}+\cdots$ $+G_{i n} x_{n} G_{i 2} \cdots G_{i n}\left|=x_{1}\right| G_{i j} \mid$. Hence

$$
W(r)=\frac{r-1}{n} \int_{\Omega}\left\{x_{1}\left|G_{i} G_{i 2} \cdots G_{i n}\right|+\cdots+x_{n}\left|G_{i 1} \cdots G_{i n-1} G_{i}\right|\right\} d \omega .
$$

\section{Columbia University,}

NEW York, N. Y. 